\title{
LOW-SENSITIVITY, LOW-POWER FOURTH-ORDER BAND-PASS ACTIVE-RC ALLPOLE FILTER USING IMPEDANCE TAPERING
}

\author{
Dražen Jurišić $^{1}$, George S. Moschytz ${ }^{2}$ and Neven Mijat ${ }^{1}$ \\ ${ }^{1}$ University of Zagreb, Department of Electronic Systems and Information Processing, Unska 3, HR-10000 \\ Zagreb,Croatia. E-mail: drazen.jurisic@,FER.hr, neven.mijat@,FER.hr \\ ${ }^{2}$ Swiss Federal Institute of Technology Zuerich (ETH), Signal and Information Processing Laboratory \\ (ISI), Sternwartstrasse 7, CH-8092 Zürich, Switzerland. E-mail: moschytz@isi.ee.ethz.ch
}

\begin{abstract}
In this paper the design of low sensitivity band-pass (BP) active resistance-capacitance $(R C)$ filters using impedance tapering is presented. $4^{\text {th }}$ order band-pass filters are considered. The design procedure for low-sensitivity low-pass (LP) prototype second-order class 4 Sallen and Key active- $R C$ allpole filters, using impedance tapering, has already been published. In this paper a low-pass to band-pass (LPBP) transformation is applied to an impedance tapered $2^{\text {nd }}$-order LP filter, and a $4^{\text {th }}$-order BP filter is constructed. The component values, selected for impedance tapering, account for the considerable decrease in sensitivity to component tolerances for the LP as well as for the BP filter. As an example, a Chebyshev $4^{\text {th }}$-order BP filter is realized, and the sensitivities are analysed. Schoeffler sensitivity measure is used as a basis for comparison, and Monte Carlo runs are performed as a double-check. A considerable improvement in sensitivity is achieved, both for the BP filter as well as well as for the original LP prototype.

Index Terms: Allpole filters, BP filters, LP-BP transformation, low-sensitivity active filters
\end{abstract}

\section{INTRODUCTION}

A procedure for the design of class-4 Sallen-and-Key [3] low-sensitivity allpole filters is presented in [1]. The class-4 filter circuit has an $R C$-ladder network in the positive feedback loop $[3,4]$. The design presented in [1] uses "impedance tapering" and it is applied to $2^{\text {nd }}$ - and $3^{\text {rd }}$-orderLP filters.

In this paper we apply impedance tapering to a $4^{\text {th }}$ order BP filter in order to decrease its sensitivity to component tolerances. The $4^{\text {th }}$-order $\mathrm{BP}$ filter is obtained applying a "lossy" LP-BP transformation [2] to a $2^{\text {nd }}$-order LP filter circuit. It is shown that the application of this transformation to a low-sensitivity, i.e. impedance tapered, $2^{\text {nd }}$-order LP filter, results by a $4^{\text {th }}$-order BP filter with low sensitivities as well. In other words sensitivity to component tolerances of the $B P$ filter is decreased in the same way as the sensitivity of the original LP prototype.

The improvement in sensitivity of a BP filter transfer function, achieved by impedance tapering, can be obtained indirectly by impedance tapering the original $2^{\text {nd }}$-order LP filter. The improvement comes with no additional cost, i.e. it requires only the selection of appropriate component values. This is demonstrated by the use of Schoeffler sensitivity measure, and double- checked using Monte Carlo analysis using PSPICE simulation.

\subsection{Impedance tapering of $\mathbf{4}^{\text {th }}$-order BP filter}

Consider the $2^{\text {nd }}$-order LP filter shown in Fig. 1. As described in [1], L-sections of the $R C$ ladder in this circuit are successively impedance-scaled upwards, from the driving source to the amplifier input. This is called "impedance tapering" and it significantly decreases the sensitivity of the filter to component tolerances. .

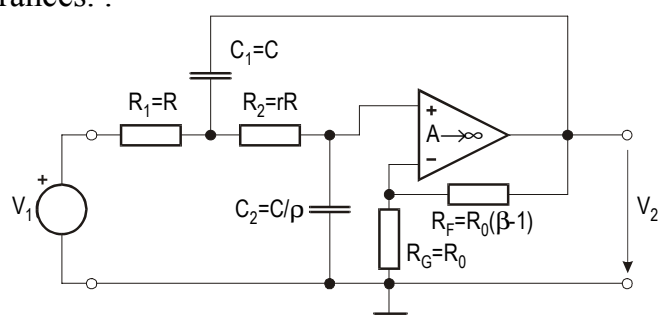

Fig. 1 Second-order LP filter $\overline{\text { circuit. }}$

The voltage transfer function $T_{L P}(s)$ for this circuit is given by

$$
T_{L P}(s)=\frac{V_{2}}{V_{1}}=\frac{K_{L P} a_{0}}{s^{2}+a_{L P 1} s+a_{L P 0}}=\frac{K_{L P} \omega_{p}^{2}}{s^{2}+\frac{\omega_{p}}{q_{p}} s+\omega_{p}^{2}} \text {.(1) }
$$

Referring to Fig. 1, we introduce impedance scaling factors $r$ and $\rho$, i.e.

$$
R_{1}=R, R_{2}=r R, C_{1}=C, C_{2}=C / \rho .
$$

In this paper we present the influence of "impedance tapering" to the fourth-order BP circuit, obtained as a result of the so-called lossy LP-BP transformation applied to an impedance-tapered $2^{\text {nd }}$-order LP filter [2]. The design procedure for the BP filter is simple, and closed-form design equations are given in [2].

The LP-BP frequency transformation doubles the filter order, thus from a $2^{\text {nd }}$-order LP filter prototype we obtain a symmetrical $4^{\text {th }}$-order BP filter. The transformation is given by:

$$
s \rightarrow \frac{s^{2}+\omega_{0}^{2}}{B s}
$$

It is shown in [2] that application of the "lossy" LP-BP transformation, which means adding a constant $\delta$ to $s$ in (3), transforms resistors in the ladder network of the LP circuit into series $R C$ circuits, and capacitors into parallel $R C$ circuits. This substitution, together with impedance scaling factors $r$ and $\rho$, is shown in Fig. 2 . 


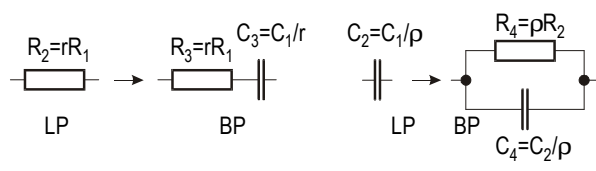

Fig. 2 Impedance scaling factors $r$ and $\rho$ by LP-BP transformation.

As a result we obtain the $4^{\text {th }}$-order BP filter shown in Fig. 3.

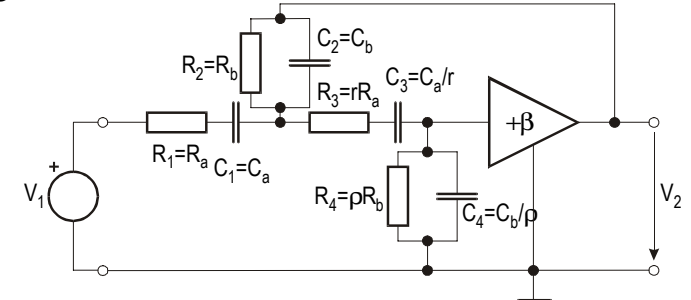

Fig. 3 Fourth-order BP filter circuit.

The voltage transfer function $T(s)$ for this circuit, expressed in terms of the coefficients $a_{i}$, is given by

$$
T_{B P}(s)=\frac{K \cdot b_{2} s^{2}}{s^{4}+a_{3} s^{3}+a_{2} s^{2}+a_{1} s+a_{0}},
$$

with

$$
\begin{gathered}
a_{0}=\omega_{0}^{4}, a_{1}=a_{3} \sqrt{a_{0}}=\omega_{p} \omega_{0}^{2} B / q_{p}, a_{2}=2 \omega_{0}^{2}+\omega_{p}^{2} B^{2}, \\
a_{3}=\omega_{p} B / q_{p}, b_{2}=\omega_{p}^{2} B^{2} .
\end{gathered}
$$

The transfer function (4) can be represented as a product of two $2^{\text {nd }}$-order transfer functions with equal pole Q, i.e. by

$$
T(s)=\frac{K_{1} \frac{\omega_{p 1}}{q} s}{s^{2}+\frac{\omega_{p 1}}{q} s+\omega_{p 1}^{2}} \cdot \frac{K_{2} \frac{\omega_{p 2}}{q} s}{s^{2}+\frac{\omega_{p 2}}{q} s+\omega_{p 2}^{2}} .
$$

The relationships between (4) and (6) is given by:

$$
\begin{array}{r}
q=\frac{q_{p} \omega_{0}}{B \omega_{p}} \sqrt{\frac{\left(4+\frac{B^{2} \omega_{p}^{2}}{\omega_{0}^{2}}\right)+\sqrt{\left(4+\frac{B^{2} \omega_{p}^{2}}{\omega_{0}^{2}}\right)^{2}-4 \frac{B^{2} \omega_{p}^{2}}{q_{p}^{2} \omega_{0}^{2}}}}{2}} \\
\frac{\omega_{p 1}}{\omega_{0}}=\frac{\omega_{0}}{\omega_{p 2}}=\frac{B q \omega_{p} / \omega_{0}+\sqrt{\left(B q \omega_{p} / \omega_{0}\right)^{2}-4 q_{p}^{2}}}{2 q_{p}}
\end{array}
$$

where $\omega_{p}$ and $q_{p}$ are pole frequency and pole Q of the $2^{\text {nd }}$-order LP prototype filter, respectively.

In the sequel we investigate the influence of impedance tapering to the sensitivity of the resulting filter. Consider, for example, a $4^{\text {th }}$-order Chebyshev BP filter with $0.5 \mathrm{~dB}$ pass-band ripple, a normalized center frequency $\omega_{0}=1$, and a normalized bandwidth $B=1$. The corresponding $2^{\text {nd }}$-order LP prototype pole parameters are $\omega_{p}=0.886021 \mathrm{rad} / \mathrm{s}$ and $q_{p}=0.863721$. Applying the frequency transformation (3) to the $2^{\text {nd }}$-order LP transfer function, we obtain, using (7) and (8), BP transfer function parameters $\omega_{p 1}=0.69486$, $\omega_{p 2}=1.43914, q=2.08029$.

Fig. 4 shows the transfer function magnitude $\alpha(\omega)=20 \log \left|T_{B P}(j \omega)\right|[\mathrm{dB}]$ of the filter circuit in Fig. 3.

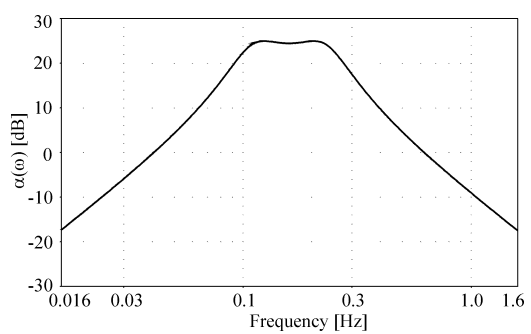

Fig. 4 Magnitude of the $4^{\text {th }}$-order BP filter.

BP filter circuit is designed using the procedure described in [2]. Various methods of impedance tapering, i.e. changing the values of the parameters $r$ and $\rho$, are applied. The resulting component values are given in Table 1. The impedance tapering is applied to the BP filter from [2], which is sensitivity optimized with respect to the "lossy" design parameter $\delta$.

\begin{tabular}{|c|c|c|c|c|c|c|c|c|}
\hline Nr. & Filter & $R_{1}$ & $C_{1}$ & $R_{2}$ & $C_{2}$ & $r$ & $\rho$ & $\beta$ \\
\hline 1) & Non Tapered & 0.605 & 1.653 & 1 & 1 & 1 & 1 & 4.80 \\
\hline 2$)$ & Impedance Tap. & 0.605 & 1.653 & 1 & 1 & 4 & 4 & 4.05 \\
\hline 3$)$ & $\mathrm{C}$ - Tapered $(r=1)$ & 1.21 & 0.827 & 1 & 1 & 1 & 4 & 2.40 \\
\hline 4$)$ & $\mathrm{R}-$ Tapered $(\rho=1)$ & 0.302 & 3.307 & 1 & 1 & 4 & 1 & 9.60 \\
\hline 5$)$ & Strongly Imp-Tap. & 0.605 & 1.653 & 1 & 1 & 10 & 10 & 3.90 \\
\hline 6$)$ & Strongly C-Tap. & 1.913 & 0.523 & 1 & 1 & 1 & 10 & 1.77 \\
\hline
\end{tabular}

Table 1 Normalized Component Values of BP Filters applying Various Tapering Techniques.

The remaining BP filter component values are:

$$
R_{3}=r R_{1}, C_{3}=C_{1} / r, R_{4}=\rho R_{2}, C_{4}=C_{2} / \rho .
$$

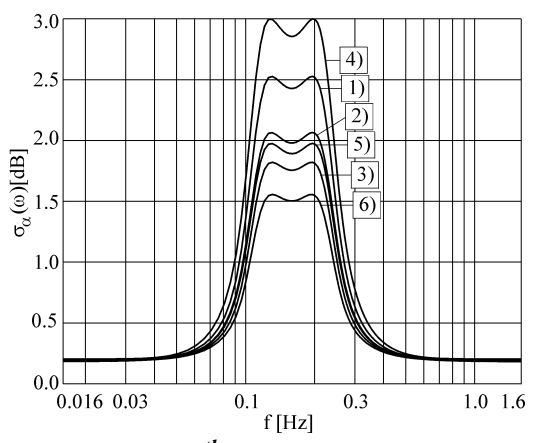

Fig. 5 Sensitivities of $4^{\text {th }}$-order BP filter circuit.

A sensitivity analysis was performed assuming the relative changes of the resistors and capacitors to be uncorrelated random variables, with a zero-mean Gaussian distribution and 1\% standard deviation. The standard deviation (which is related to the Shoeffler sensitivities) of the variation of the logarithmic gain $\Delta \alpha=8.68588 \Delta\left|T_{B P}(\omega)\right| /\left|T_{B P}(\omega)\right|$, with respect to the passive elements, is calculated for the filter examples in Table 1 and shown in Fig. 5.

Observing the standard deviation $\sigma_{\alpha}(\omega)[\mathrm{dB}]$ of the variation of the logarithmic gain $\Delta \alpha$ in Fig. 5 we conclude that the ideally impedance-tapered filter (No. 2) has considerably decreased sensitivities, compared to the non-tapered standard circuit version (No. 1). By tapering only the capacitors, while keeping the resistor values equal ( $r=1)$, (No. 3), the filter sensitivities are decreased even more. The resistively tapered filter (No. 4) has the highest sensitivities, for the reason explained 
below. Furthermore, a strong ideally-tapered filter circuit (No. 5), with a tapering factor $r=\rho=10$, has higher sensitivites than the capacitively tapered filter (No. 3) with a medium capacitive scaling factor $\rho=4$, and equal resistors. Thus the best results are obtained with capacitivelly tapered filters and equal resistors (circuits No. 3 and 6).

The reason for the lower sensitivity, of the equal resistors and tapered capacitors case, is the same here, for the $4^{\text {th }}$-order BP case, as for the $2^{\text {nd }}$-order LP in [1]. In both cases the expressions for the coefficient-tocomponent sensitivities $S_{x}^{a_{i}}$ are partially proportional ${ }^{\dagger}$ to the resistive scaling factor $r$ but exclusively inversely proportional to the capacitive scaling factor $\rho$. Thus, the coefficient-to-component sensitivities can be significantly reduced by choosing $r=1$ and increasing only $\rho$. In fact, in all cases, capacitive tapering with equal resistors $(r=1)$ results by a stronger desensitisation than ideal impedance tapering $(r=\rho)$.

Monte Carlo runs, carried out for our example, shown in Fig. 9, confirm the above conclusions.

In summary, for the general $4^{\text {th }}$-order allpole Class-4 $B P$ filter designed by means of lossy LP-BP transformation, ideal impedance tapering provides low sensitivity circuits. Capacitive impedance tapering with equal resistors $(\rho=1)$, provides circuits with the lowest sensitivity to the component tolerances of the circuit.

\section{COMPARISON OF $4^{\text {th }}-$ ORDER FILTER WITH 2-BIQUAD CASCADE}

In this section we compare the performance of our new, single operational amplifier, $4^{\text {th }}$-order BP filter to a cascade of two $2^{\text {nd }}$-order BP filter "biquads" as shown in Fig. 6. They are compared with respect to sensitivity to component tolerances, and to thermal output noise.

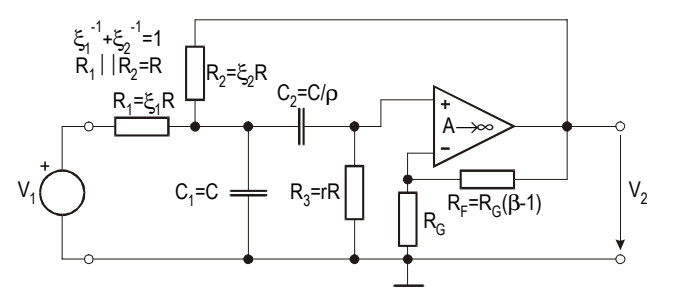

(a)

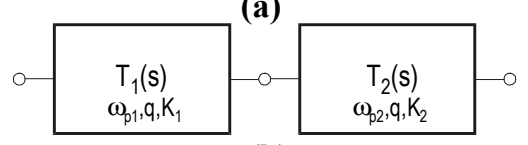

(b)

Fig. 6 (a) $2^{\text {nd }}$-order BP filter block. (b) BP Cascade.

We apply impedance tapering, to minimize sensitivity, to both the single-amplifier (1-OA) filter and to the two biquads in cascade (CAS).

The circuit in Fig. $6 \mathrm{a}$ is a $2^{\text {nd }}$-order BP filter type B [6] with positive feedback, i.e. class 4 circuit $[4,5]$.

$\dagger$ By "partial proportionality" we mean that $r$ will appear partially in the numerator, partially in the denominator of the sensitivity expressions.
The normalized component values for the two biquads are calculated, and given in Table 2.

\begin{tabular}{|c|c|c|c|c|c|c|c|c|}
\hline Nr. & Filter-Blocks) & $R$ & $C$ & $\xi_{1}$ & $r$ & $\rho$ & $\beta$ \\
\hline \multirow{2}{*}{$1)$} & \multirow{2}{*}{ Non Tapered } & $1)$ & 0.6949 & 1 & 2 & 1 & 1 & 5.0386 \\
\cline { 3 - 8 } & & 2) & 1.4391 & 1 & 2 & 1 & 1 & 5.0386 \\
\hline \multirow{2}{*}{ 2) } & Impedance & $1)$ & 0.6949 & 1 & 2 & 4 & 4 & 3.5386 \\
\cline { 3 - 9 } & Tapered & 2) & 1.4391 & 1 & 2 & 4 & 4 & 3.5386 \\
\hline
\end{tabular}

Table 2 Component Values of $2^{\text {nd }}$-order BP Sections.

The remaining component values follow from

$$
R_{1}=\xi_{1} R, R_{2}=\xi_{2} R, R_{3}=r R, C_{1}=C, C_{2}=C / \rho .
$$

using tapering factors as presented in Table 2 .

The sensitivities for both the 1-OA and CAS $4^{\text {th }}$-order BP filters, are presented in Fig. 7, where "T" stands for tapered.

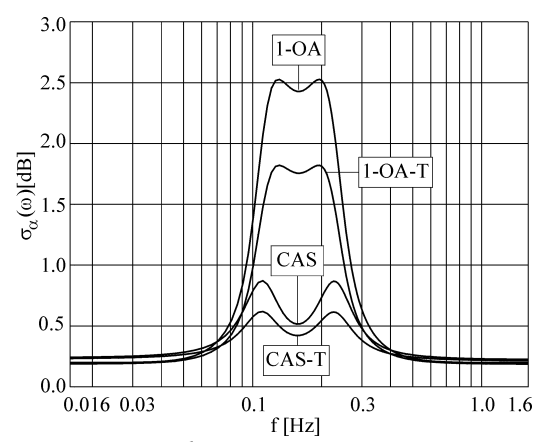

Fig. 7 Sensitivities of $4^{\text {th }}$-order BP filter circuits.

To investigate the noise performance, we perform a simulation of output noise with PSPICE, using the Texas Instruments TL081 TX as the operational amplifier model. For this purpose the real values of resistors and capacitors are calculated, using the denormalization procedure

$$
R=R_{0} \cdot R_{n}, C=\frac{C_{n}}{\omega_{0} R_{0}},
$$

where $\omega_{0}=2 \pi \cdot f_{0}$ is the center frequency of the BP,and $R_{0}$ is the denormalisation impedance.

As an example, for the center frequency $f_{0}=1 \mathrm{kHz}$, and the capacitors value $C=5 \mathrm{nF}$, we obtain the resistance $R_{0}=1 /\left(2 \pi \cdot 10^{3} \cdot 5 \cdot 10^{-9}\right)=31.831 \mathrm{k} \Omega$. Using (11) the denormalized component values for circuits Nr.1) "Non Tapered" and Nr.3) "C-Tapered $(r=1)$ " are obtained from Table 1, for the 1-OA BP filters, and for the CAS BP filters from Table 2 . We chose $R_{G}=10 \mathrm{k} \Omega$.

The noise characteristics, for both 1-OA and CAS BP filters, are presented in Fig. 8.

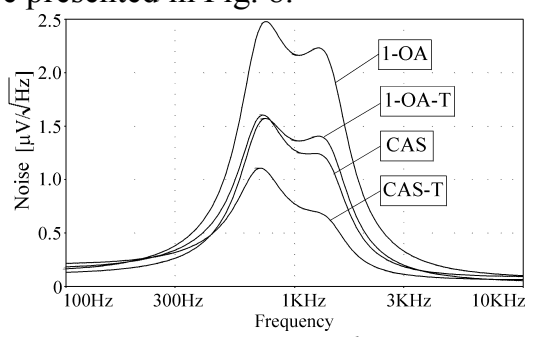

Fig. 8 Noise spectral density of $4^{\text {th }}$-order BP filters.

From Figs. 7 and 8 we conclude that the fourth-order BP filter with single operational amplifier (1-OA), which is capacitivelly tapered with equal resistors values $(r=1)$, has worse performance, both with respect 
to the sensitivity and thermal output noise, then a cascade of two second-order BP filter blocks. However, the Figs. 7 and 8 also confirm that impedance tapering improves filter performances from both aspects. Thus our new single operational amplifier (1-OA) is a reasonably good filter in the sense of sensitivity to component tolerances and noise performances if we
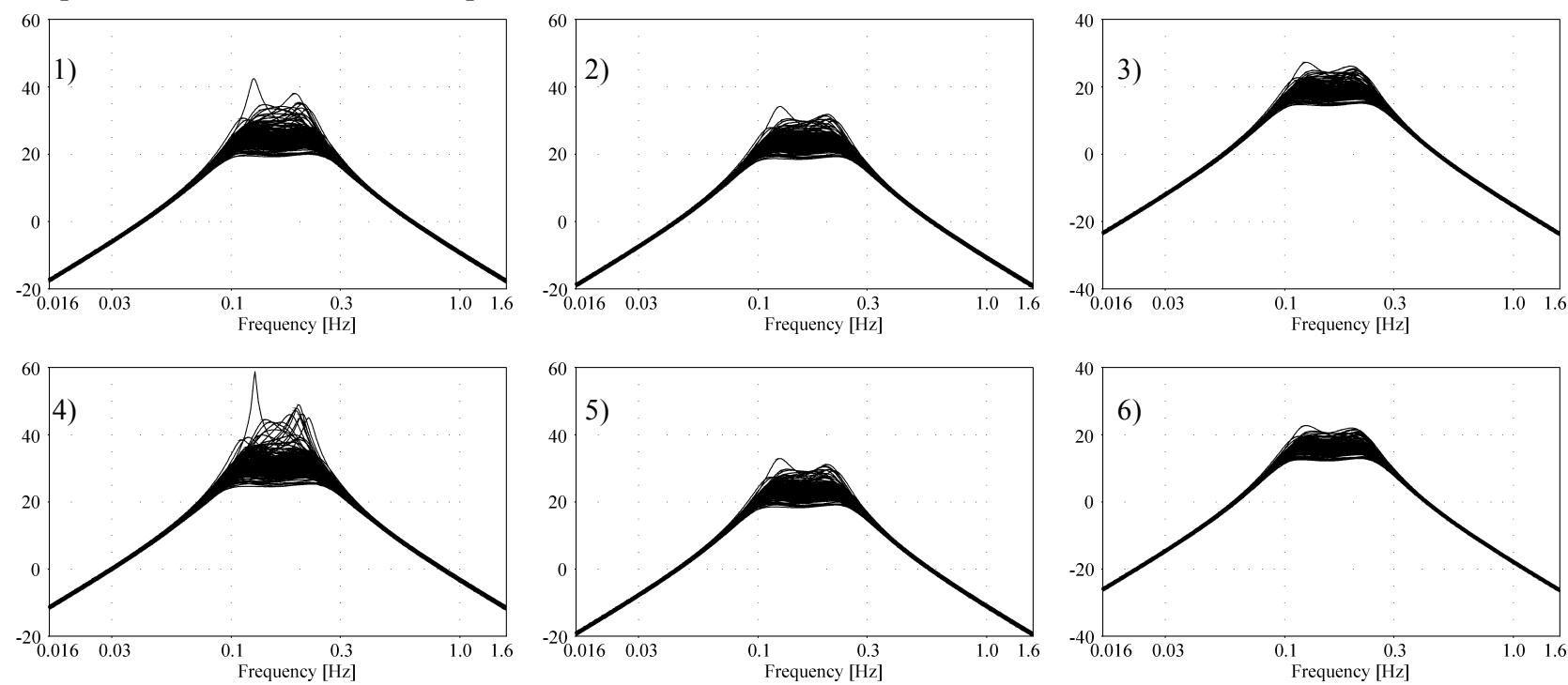

wish to minimize power by halving the number of opamps, and significantly reduce the passive components count. In any event, capacitive impedance tapering with equal resistors $(\rho=1)$, provides circuits with lower noise and lower sensitivity to component tolerances than that of non-tapered circuits.

Fig. 9 Results of Monte Carlo runs carried out on 4th-order BP filter with $\omega_{0}=1$ and $B=1$.

\section{CONCLUSIONS}

A procedure for the design of allpole low-sensitivity, low-power $4^{\text {th }}$-order BP active $R C$ filters using impedance tapering is presented. The filters are constructed using a LP-BP (LP-BP) transformation [2]. The filter uses only one operational amplifier, and a minimum number of passive components. The design procedure using impedance tapering adds nothing to the cost of conventional circuits; component count and topology remains the same. Using the appropriate impedance tapering factors $r$ and $\rho$, we can influence the sensitivity to component tolerances. Whichever tapering method is used, the circuit performance is improved with regard to thermal output noise and sensitivity. The improvement in sensitivity of the BP filter is the same as that of the corresponding LP prototype filter [1]. Ideal impedance tapering invariably provides circuits with low sensitivity and low noise. However, for reasons related to the filter topology, capacitivelly tapered circuits with equal resistors $(\rho=1)$ have minimum sensitivity to component tolerances. Furthermore, the reduction in power and component count achieved with the single-amplifier fourth-order BP filters is obtained at a price: a cascade of two impedance-tapered biquads is better on both counts. Thus the decision on which way to go is typically one of tradeoffs: low power and component count versus 